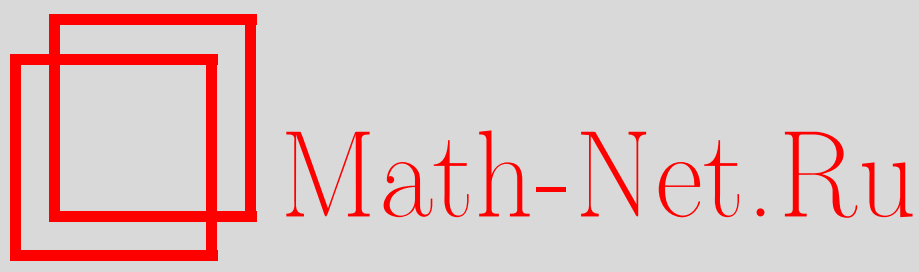

Г. Г. Магарил-Ильяев, К. Ю. Осипенко, Оптимальное восстановление значений функций и их производных по неточно заданному преобразованию Фурье, Матем. сб., 2004, том 195, номер 10, 67-82

DOI: https://doi.org/10.4213/sm853

Использование Общероссийского математического портала Math-Net.Ru подразумевает, что вы прочитали и согласны с пользовательским соглашением

http://www . mathnet.ru/rus/agreement

Параметры загрузки:

IP: 52.23 .180 .231

26 апреля 2023 г., 05:14:18 
УДК 517.5

Г.Г. Магарил-Ильяев, К. Ю. Осипенко

\title{
Оптимальное восстановление значений функций и их производных по неточно заданному преобразованию Фурье
}

\begin{abstract}
В работе рассматриваются задачи оптимального восстановления значения производных функций по информации о преобразовании Фурье этих функций, заданном приближенно на конечном интервале или всей прямой. Изучается также тесно связанная с этой проблематикой задача С.Б. Стечкина о приближении производных ограниченньми линейными функционалами. Получены соответствующие этим постановкам точные неравенства для производных колмогоровского типа.

Библиография: 15 названия.
\end{abstract}

\section{§1. Постановка задач}

Начнем с формулировки конкретных задач, которые изучаются в настоящей работе, а затем приведем обшую постановку задачи оптимального восстановления функционалов, объединяюшую эти задачи. Пусть $S$-пространство Шварца быстро убываюших бесконечно дифференцируемых функций на $\mathbb{R}, S^{\prime}$ - соответствующее пространство обобщенных функций, $F: S^{\prime} \rightarrow S^{\prime}$ - преобразование Фурье, $n \in \mathbb{N}$ и $1 \leqslant p \leqslant \infty$. Положим

$$
X_{p}^{n}=\left\{x \in S^{\prime} \mid F x(\cdot) \in L_{p}(\mathbb{R}), x^{(n)}(\cdot) \in L_{2}(\mathbb{R})\right\}
$$

И

$$
C_{p}^{n}=\left\{x(\cdot) \in X_{p}^{n} \mid\left\|x^{(n)}(\cdot)\right\|_{L_{2}(\mathbb{R})} \leqslant 1\right\} .
$$

Задача об оптимальном восстановлении значения $x^{(k)}(\tau), 0 \leqslant k<n, \tau \in \mathbb{R}$, на классе $C_{p}^{n}$ по информации о преобразовании Фурье $F x(\cdot)$, заданном на интервале $\Delta_{\sigma}=(-\sigma, \sigma), 0<\sigma \leqslant \infty$, с погрешностью $\delta>0$ в метрике пространства $L_{p}\left(\Delta_{\sigma}\right)$, заключается в нахождении величины

$$
E_{p}(n, k, \sigma, \delta)=\inf _{\varphi} \sup _{\substack{x(\cdot) \in C_{p}^{n}, y(\cdot) \in L_{p}\left(\Delta_{\sigma}\right) \\\|F x(\cdot)-y(\cdot)\|_{L_{p}\left(\Delta_{\sigma}\right)} \leqslant \delta}}\left|x^{(k)}(\tau)-\varphi(y(\cdot))\right|
$$

Работа выполнена при финансовой поддержке Российского фонда фундаменталњных исследований (гранты №№ 02-01-39012, 02-01-00386), Программы поддержки ведущих научных школ РФ (грант № НШ-304.2003.1), Программы "Университеты России" (грант № УР.04.03.067), а также при поддержке U.S.CRDF-R.F.Ministry of Education Award (грант № VZ-0100-0). 
(где нижняя грань берется по всем функциям $\varphi: L_{p}\left(\Delta_{\sigma}\right) \rightarrow \mathbb{C}$ ), называемой погрешностью оптимального восстановления, и функции $\widehat{\varphi}$, на которой достигается нижняя грань в (1), называемой оптимальным методом восстановления.

В работе изучается также задача о наилучшем приближении $x^{(k)}(\tau), 0 \leqslant k<n$, $\tau \in \mathbb{R}$, на классе $C_{p}^{n}$ по информации о преобразовании Фурье $F x(\cdot)$, заданном на интервале $\Delta_{\sigma}=(-\sigma, \sigma)$, линейными непрерывными функционалами на $L_{p}\left(\Delta_{\sigma}\right)$, норма которых не превосходит некоторого фиксированного положительного числа $N$. Она состоит в нахождении величины

$$
e_{p}(n, k, \sigma, N)=\inf _{y^{*}} \sup _{x(\cdot) \in C_{p}^{n}}\left|x^{(k)}(\tau)-\left\langle y^{*}, F x(\cdot)\right\rangle\right|
$$

(где нижняя грань берется по всем линейным функционалам $y^{*}$ на $L_{p}\left(\Delta_{\sigma}\right)$ таким, что $\left.\left\|y^{*}\right\| \leqslant N\right)$, а также функционала $\widehat{y}^{*}$, на котором достигается нижняя грань в (2), назьваемого әкстремальным.

Если в (2) вместо $F x(\cdot)$ поставить $x(\cdot)$, то мы получаем классическую задачу С. Б. Стечкина, так что (2) есть некоторое ее обобщение, которое мы также называем задачей Стечкина.

Приведем теперь общую постановку задачи об оптимальном восстановлении линейного функционала на классе элементов по некоторой информации о самих элементах. Пусть $X$ - вешественное или комплексное векторное пространство и $C$ непустое подмножество (класс элементов) в $X$. Про каждый элемент $x \in C$ мы располагаем информацией $I(x)$, где $I$ - отображение (назьваемое информационным) из $C$ в другое вещественное или комплексное векторное пространство $Y$. В случае, когда информация задана неточно, $I$ - многозначное отображение. Пусть, далее, заданы линейный функционал $x^{\prime}$ на $X$ и семейство $\Phi$ функций $\varphi: Y \rightarrow \mathbb{R}(\mathbb{C})$. Задача об оптимальном восстановлении функционала $x^{\prime}$ на классе $C$ по информации $I$ с помощью функций (методов восстановления) из $\Phi$ заключается в нахождении величины

$$
E\left(x^{\prime}, C, I\right)=\inf _{\varphi \in \Phi} \sup _{\substack{x \in C \\ y \in I(x)}}\left|\left\langle x^{\prime}, x\right\rangle-\varphi(y)\right|,
$$

называемой погрешностью оптимального восстановления (функционала $x^{\prime}$ на $C$ по информации $I)$, и метода, на котором достигается нижняя грань в (3), называемого оптимальным методом восстановления.

Задачи (1) и (2) укладываются в общую схему. В первом случае $X=X_{p}^{n}$, $C=C_{p}^{n}, Y=L_{p}\left(\Delta_{\sigma}\right), I: X_{p}^{n} \rightarrow L_{p}\left(\Delta_{\sigma}\right), I x(\cdot)=\left.F x(\cdot)\right|_{\Delta_{\sigma}}+\delta B L_{p}\left(\Delta_{\sigma}\right)$, где $B L_{p}\left(\Delta_{\sigma}\right)$ - единичный шар в $L_{p}\left(\Delta_{\sigma}\right),\left\langle x^{\prime}, x(\cdot)\right\rangle=x^{(k)}(\tau)$ и $\Phi-$ совокупность всех функций на $L_{p}\left(\Delta_{\sigma}\right)$. Для второй задачи $X=X_{p}^{n}, C=C_{p}^{n}, Y=L_{p}\left(\Delta_{\sigma}\right)$, $I: X_{p}^{n} \rightarrow L_{p}\left(\Delta_{\sigma}\right), \quad I x(\cdot)=\left.F x(\cdot)\right|_{\Delta_{\sigma}},\left\langle x^{\prime}, x(\cdot)\right\rangle=x^{(k)}(\tau)$ и $\Phi=N B Y^{*}$, где $B Y^{*}$ - единичный шар в сопряженном пространстве к $Y$.

Задача оптимального восстановления линейного функционала на классе элементов для случая, когда $I$ - линейное отображение, $\operatorname{dim} Y<\infty$ и $\Phi-$ множество всех функций из $Y$ в $\mathbb{R}$, была поставлена С. А. Смоляком [1]. Им было доказано, что если в этой ситуации $C$ - вьпуклое центрально симметричное множество, то среди оптимальных методов есть линейньй. Далее эта задача обобшалась и развивалась в различных направлениях (см. [2]-[7]). 
Задачи оптимального восстановления функций и их производных в метрике $L_{2}$ (т.е. задача оптимального восстановления оператора, а не функционала) по неточно заданным коэффициентам Фурье (для периодических функций) и по неточно заданному преобразованию Фурье (для функций на прямой) изучались в работах [8], [9]. Круг проблем, связанных с задачей Стечкина, освешен в обзорной статье [10].

Аналог задачи (1) в периодическом случае при $p=\infty$ рассматривался в работе [11].

\section{§2. Формулировки основных результатов}

В силу инвариантности рассматриваемых классов относительно сдвига всюду в дальнейшем считаем, что $\tau=0$. Начнем со случая, когда $p=\infty$.

TeOpema 1. Пусть $\delta>0, k, n \in \mathbb{Z}, 0 \leqslant k<n, 0<\sigma \leqslant \infty$,

$$
\widehat{\sigma}=\left(\frac{\pi(2 n+1)(2 n-2 k-1)}{2 \delta^{2}(2 n-k)}\right)^{1 /(2 n+1)}
$$

$u \sigma_{0}=\min \{\sigma, \widehat{\sigma}\}$. Тогдa

$$
E_{\infty}(n, k, \sigma, \delta)=\frac{\sigma_{0}^{k+1}}{\pi}\left(\frac{\delta}{k+1}+\sqrt{\frac{1}{2 n-2 k-1}\left(\frac{\pi}{\sigma_{0}^{2 n+1}}-\frac{\delta^{2}}{2 n+1}\right)}\right)
$$

а метод

$$
\widehat{\varphi}(y(\cdot))=\frac{1}{2 \pi} \int_{|t|<\sigma_{0}}(i t)^{k}\left(1-\delta \lambda|t|^{2 n-k}\right) y(t) d t
$$

әде

$$
\lambda=\frac{\sigma_{0}^{-2 n+k}}{\sqrt{2 n-2 k-1}}\left(\frac{\pi}{\sigma_{0}^{2 n+1}}-\frac{\delta^{2}}{2 n+1}\right)^{-1 / 2},
$$

является оптимальньц.

Из теоремы 1 вытекает, что при $\sigma \geqslant \widehat{\sigma}$

$$
E_{\infty}(n, k, \sigma, \delta)=K \delta^{(2 n-2 k-1) /(2 n+1)},
$$

где

$$
K=\frac{(n+1 / 2)^{(k+1) /(2 n+1)}}{k+1}\left(\frac{2 n-k}{\pi(2 n-2 k-1)}\right)^{(2 n-k) /(2 n+1)} .
$$

Тем самым в рассматриваемой задаче имеет место эффект "насьщения" погрешности оптимального восстановления, заключающийся в том, что при фиксированном $\delta>0$ знание преобразования Фурье функции из $C_{\infty}^{n}$, заданного с погрешностью $\delta$ в равномерной метрике, на интервалах, больших чем $\Delta_{\widehat{\sigma}}$, не ведет к уменьшению погрешности оптимального восстановления. Таким образом, нарушение соотношения

$$
\delta^{2} \sigma^{2 n+1} \leqslant \frac{\pi(2 n+1)(2 n-2 k-1)}{2(2 n-k)}
$$


приводит к тому, что получаемая информация о преобразовании Фурье оказывается избыточной. Этот факт нам представляется важньм для приложений, когда нужно считаться с тем, что получение дополнительной информации требует определенных затрат.

Из равенства (6) в силу инвариантности пространства $X_{\infty}^{n}$ относительно сдвига вытекает следующий результат.

СлЕДСТВИЕ 1. Пусть $k, n \in \mathbb{Z} u 0 \leqslant k<n$. Тогда имеет место точное неравенство

$$
\left\|x^{(k)}(\cdot)\right\|_{L_{\infty}(\mathbb{R})} \leqslant K\|F x(\cdot)\|_{L_{\infty}(\mathbb{R})}^{(2 n-2 k-1) /(2 n+1)}\left\|x^{(n)}(\cdot)\right\|_{L_{2}(\mathbb{R})}^{(2 k+2) /(2 n+1)}
$$

әде константа $K$ определена равенством (7).

Перейдем теперь к задаче (1) для $p=1$. Если $k>0$ и $\sigma<\infty$, положим

$$
\Phi(\varepsilon)=\frac{4 \pi}{\sigma^{2 n-1}} \frac{\varepsilon^{2(2 n-k-1)}\left(\int_{1}^{\varepsilon}\left(x^{k}-1\right) x^{-2 n} d x\right)^{2}}{\varepsilon^{2 n-2 k-1} \int_{1}^{\varepsilon}\left(x^{k}-1\right)^{2} x^{-2 n} d x+(2 n-2 k-1)^{-1}}
$$

(здесь и далее для краткости записи не приводятся выражения для интегралов, которые могут быть явно вычислены). Очевидно, что функция $\Phi(\cdot)$ непрерьвна на $(1,+\infty)$. Нетрудно убедиться, что $\Phi(\varepsilon) \rightarrow 0$ при $\varepsilon \rightarrow 1$ и $\Phi(\varepsilon) \rightarrow+\infty$ при $\varepsilon \rightarrow+\infty$. Тем самым для любого $\delta>0$ уравнение

$$
\Phi(\varepsilon)=\delta^{2}
$$

имеет решение, принадлежащее интервалу $(1,+\infty)$.

Теорема 2. Пусть $\delta>0, k, n \in \mathbb{N}, \quad 0<k<n, \quad 0<\sigma \leqslant \infty$. Положим

$$
\begin{aligned}
& a= \begin{cases}\frac{\sigma}{\varepsilon_{\delta}}, & 0<\sigma<\infty \\
\left(\frac{2 \pi(2 n-2 k-1)}{\delta^{2}(2 n-1)(2 n-k-1)}\right)^{1 /(2 n-1)}, & \sigma=\infty\end{cases} \\
& \lambda= \begin{cases}\frac{2}{\delta}\left(\frac{\varepsilon_{\delta}}{\sigma}\right)^{2 n-k-1} \int_{1}^{\varepsilon_{\delta}}\left(x^{k}-1\right) x^{-2 n} d x, & 0<\sigma<\infty ; \\
\frac{k \delta^{(2 n-2 k-1) /(2 n-1)}}{\pi(2 n-2 k-1)}\left(\frac{2 \pi(2 n-2 k-1)}{(2 n-1)(2 n-k-1)}\right)^{k /(2 n-1)}, & \sigma=\infty,\end{cases}
\end{aligned}
$$

где $\varepsilon_{\delta}-$ решение уравнения (9). Тогда

$$
E_{1}(n, k, \sigma, \delta)=\lambda+\frac{\delta}{2 \pi} a^{k}
$$

а метод

$$
\widehat{\varphi}(y(\cdot))=\frac{1}{2 \pi} \int_{|t|<\sigma} \mu_{\delta}(t) y(t) d t
$$


əде

$$
\mu_{\delta}(t)= \begin{cases}(i t)^{k}, & |t| \leqslant a \\ (i a)^{k} \operatorname{sign} t^{k}, & a<|t|<\sigma,\end{cases}
$$

является оптимальныц. При $k=0$

$$
E_{1}(n, 0, \sigma, \delta)=\frac{\delta}{2 \pi}+\frac{1}{\sigma^{n-1 / 2} \sqrt{\pi(2 n-1)}}
$$

u метод

$$
\widehat{\varphi}(y(\cdot))=\frac{1}{2 \pi} \int_{|t|<\sigma} y(t) d t
$$

оптимальный.

Из теоремы 2 при $\sigma=\infty$ вытекает

СЛЕДСТвИЕ 2 . Пусть $k, n \in \mathbb{Z} u 0 \leqslant k<n$. Тогда имеет место точное неравенство

$$
\left\|x^{(k)}(\cdot)\right\|_{L_{\infty}(\mathbb{R})} \leqslant K_{1}\|F x(\cdot)\|_{L_{1}(\mathbb{R})}^{(2 n-2 k-1) /(2 n-1)}\left\|x^{(n)}(\cdot)\right\|_{L_{2}(\mathbb{R})}^{k /(2 n-1)},
$$

2дe

$$
K_{1}=\frac{1}{(2 n-k-1)^{k /(2 n-1)}}\left(\frac{2 n-1}{2 \pi(2 n-2 k-1)}\right)^{(2 n-k-1) /(2 n-1)} .
$$

Перейдем к случаю $p=2$. Если $\sigma<\infty$, то положим

$$
\Psi(h)=\frac{2 \pi \sigma^{2 n-2 k-1} h^{4 n-2 k-1} \int_{0}^{\sigma h} x^{2 k}\left(1+x^{2 n}\right)^{-2} d x}{(\sigma h)^{2 n-2 k-1} \int_{0}^{\sigma h} x^{2(n+k)}\left(1+x^{2 n}\right)^{-2} d x+(2 n-2 k-1)^{-1}} .
$$

Нетрудно убедиться, что функция $\Psi(\cdot)$ непрерывна на $(0,+\infty), \Psi(h) \rightarrow 0$ при $h \rightarrow 0$ и $\Phi(h) \rightarrow+\infty$ при $h \rightarrow+\infty$. Тем самым для любого $\delta>0$ уравнение

$$
\Psi(h)=\delta^{2}
$$

имеет решение, принадлежашее интервалу $(0,+\infty)$.

Tеорема 3. Пусть $\delta>0, k, n \in \mathbb{Z}, 0 \leqslant k<n, 0<\sigma \leqslant \infty$. Если $0<\sigma<\infty$, то через $h_{\delta}$ обозначим решение уравнения (13), а если $\sigma=\infty$, то положим

$$
h_{\delta}=\left(\frac{(2 k+1) \delta^{2}}{2 \pi(2 n-2 k-1)}\right)^{1 /(2 n)} .
$$

Тогда

$$
E_{2}(n, k, \sigma, \delta)=\frac{\delta^{2}+2 \pi h_{\delta}^{2 n}}{2 \pi \delta h_{\delta}^{k+1 / 2}}\left(2 \int_{0}^{\sigma h_{\delta}} x^{2 k}\left(1+x^{2 n}\right)^{-2} d x\right)^{1 / 2}
$$


а метод

$$
\widehat{\varphi}(y(\cdot))=\frac{1}{2 \pi} \int_{|t|<\sigma} \frac{(i t)^{k}}{1+\left(h_{\delta} t\right)^{2 n}} y(t) d t
$$

является оптимальным.

При $\sigma=\infty$ получаем

$$
E_{2}(n, k, \infty, \delta)=K_{2} \delta^{(2 n-2 k-1) /(2 n)},
$$

где

$$
K_{2}=\left((2 k+1) \sin \frac{\pi(2 k+1)}{2 n}\right)^{-1 / 2}\left(\frac{2 k+1}{2 \pi(2 n-2 k-1)}\right)^{(2 n-2 k-1) /(4 n)}
$$

Отсюда следует точное неравенство

$$
\left\|x^{(k)}(\cdot)\right\|_{L_{\infty}(\mathbb{R})} \leqslant K_{2}\|F x(\cdot)\|_{L_{2}(\mathbb{R})}^{(2 n-2 k-1) /(2 n)}\left\|x^{(n)}(\cdot)\right\|_{L_{2}(\mathbb{R})}^{(2 k+1) /(2 n)} .
$$

В силу равенства Парсеваля

$$
\|F x(\cdot)\|_{L_{2}(\mathbb{R})}=\sqrt{2 \pi}\|x(\cdot)\|_{L_{2}(\mathbb{R})}
$$

оно может быть записано в виде

$\left\|x^{(k)}(\cdot)\right\|_{L_{\infty}(\mathbb{R})} \leqslant(2 \pi)^{(2 n-2 k-1) /(4 n)} K_{2}\|x(\cdot)\|_{L_{2}(\mathbb{R})}^{(2 n-2 k-1) /(2 n)}\left\|x^{(n)}(\cdot)\right\|_{L_{2}(\mathbb{R})}^{(2 k+1) /(2 n)}$.

Это неравенство было доказано Л. В. Тайковым [12].

Перейдем теперь к задаче Стечкина о приближении $k$-й производной функции из класса $C_{p}^{n}$ по информации о ее преобразовании Фурье на интервале $\Delta_{\sigma}$ с помощью линейных функционалов, норма которых не превосходит фиксированного положительного числа $N$.

Tеорема 4. Пусть $n, k \in \mathbb{Z}, 0 \leqslant k<n, \quad 0<\sigma \leqslant \infty, \quad N>0$,

$$
\widehat{\sigma}_{N}=\left(\frac{\pi N(k+1)(2 n+1)}{2 n-k}\right)^{1 /(k+1)}
$$

$u \sigma_{N}=\min \left\{\sigma, \widehat{\sigma}_{N}\right\}$. Тогдa

$$
e_{\infty}(n, k, \sigma, N)=\frac{\sigma_{N}^{-n+k+1 / 2}}{\sqrt{\pi}} \sqrt{\frac{1}{2 n-2 k-1}+\frac{\gamma^{2}(\sigma, N)}{2 n+1}},
$$

əдe

$$
\gamma(\sigma, N)=\max \left\{0,(2 n+1)\left(\frac{1}{k+1}-\frac{\pi N}{\sigma_{N}^{k+1}}\right)\right\}
$$

и функционал

$$
\left\langle\widehat{y}^{*}, F x(\cdot)\right\rangle=\frac{1}{2 \pi} \int_{|t|<\sigma_{N}}(i t)^{k}\left(1-\gamma(\sigma, N)\left(\frac{|t|}{\sigma_{N}}\right)^{2 n-k}\right) F x(t) d t
$$


является әкстремальным.

Из теоремы 4 вытекает, что при фиксированном $N$ для $\sigma \geqslant \widehat{\sigma}_{N}$

$$
\begin{aligned}
& e_{\infty}(n, k, \sigma, N) \\
& \quad=\sqrt{\frac{2 k+2}{2 n-2 k-1}}\left(\frac{2 n-k}{\pi(k+1)(2 n+1)}\right)^{(2 n-k) /(2 k+2)} N^{-(2 n-2 k-1) /(2 k+2)} .
\end{aligned}
$$

Это означает, что аналогично задаче восстановления в рассматриваемой задаче Стечкина также наблюдается эффект "насыщения", заключающийся в том, что при фиксированном $N$ знание преобразования Фурье на интервалах, больших чем $\left(-\widehat{\sigma}_{N}, \widehat{\sigma}_{N}\right)$, не ведет к уменьшению погрешности $e_{\infty}(n, k, \sigma, N)$. Аналогом соотношения (8) здесь является неравенство

$$
\frac{\sigma^{k+1}}{N} \leqslant \frac{\pi(k+1)(2 n+1)}{2 n-k}
$$

нарушение которого ведет к избыточности получаемой информации о преобразовании $Ф$ урье.

Tеорема 5. Пусть $n, k \in \mathbb{N}, 0<k<n u N>0$. Тогда при $\sigma<\infty$

$$
\begin{aligned}
& e_{1}(n, k, \sigma, N)=\frac{1}{\sqrt{\pi} \sigma^{n-k-1 / 2}} \\
& \quad \times \sqrt{\frac{2 k^{2} \varepsilon^{2 n-2 k-1}}{(2 n-2 k-1)(2 n-k-1)(2 n-1)}+\frac{2 \varepsilon^{-k}}{2 n-k-1}-\frac{\varepsilon^{-2 k}}{2 n-1}},
\end{aligned}
$$

$2 \partial e$

$$
\varepsilon=\frac{\sigma}{\left(2 \pi N_{0}\right)^{1 / k}}, \quad N_{0}=\min \left\{N, \frac{\sigma^{k}}{2 \pi}\right\}
$$

и функиионал

$$
\begin{aligned}
\left\langle\widehat{y}^{*}, F x(\cdot)\right\rangle=\frac{1}{2 \pi} & \int_{|t|<\left(2 \pi N_{0}\right)^{1 / k}}(i t)^{k} F x(t) d t \\
& +N_{0} i^{k} \int_{\left(2 \pi N_{0}\right)^{1 / k} \leqslant|t|<\sigma} \operatorname{sign} t^{k} F x(t) d t
\end{aligned}
$$

является әкстремальным. При $\sigma=\infty$

$$
e_{1}(n, k, \infty, N)=\frac{\sqrt{2} k(2 \pi N)^{-(2 n-2 k-1) /(2 k)}}{\sqrt{\pi(2 n-1)(2 n-k-1)(2 n-2 k-1)}},
$$

а функционал

$$
\left\langle\widehat{y}^{*}, F x(\cdot)\right\rangle=\frac{1}{2 \pi} \int_{|t|<(2 \pi N)^{1 / k}}(i t)^{k} F x(t) d t+N \int_{|t| \geqslant(2 \pi N)^{1 / k}} i^{k} \operatorname{sign} t^{k} F x(t) d t
$$


экстремальный. Если $k=0$, то

при этом функиионал

$$
e_{1}(n, 0, \sigma, N)= \begin{cases}\infty, & 0<N<\frac{1}{2 \pi} ; \\ \frac{1}{\sigma^{n-1 / 2} \sqrt{\pi(2 n-1)}}, & N \geqslant \frac{1}{2 \pi}, \sigma<\infty ; \\ 0 & N \geqslant \frac{1}{2 \pi}, \sigma=\infty,\end{cases}
$$

$$
\left\langle\widehat{y}^{*}, F x(\cdot)\right\rangle=\frac{1}{2 \pi} \int_{|t|<\sigma} F x(t) d t
$$

является әкстремальным.

Рассмотрим теперь задачу Стечкина для случая, когда $p=2$. Положим

$$
\Omega(h)=\frac{1}{2 \pi^{2} h^{2 k+1}} \int_{0}^{\sigma h} x^{2 k}\left(1+x^{2 n}\right)^{-2} d x .
$$

Функция $\Omega(h)$ непрерьвна при $h \in(0,+\infty)$. При этом

$$
\lim _{h \rightarrow 0} \Omega(h)=\frac{\sigma^{2 k+1}}{2 \pi^{2}(2 k+1)},
$$

$\operatorname{aim}_{h \rightarrow \infty} \Omega(h)=0$. Поэтому при всех

уравнение

$$
0<N<\frac{\sigma^{k+1 / 2}}{\pi \sqrt{2(2 k+1)}}
$$

$$
\Omega(h)=N^{2}
$$

имеет решение.

Teоpema 6. Пусть $n, k \in \mathbb{Z}, 0 \leqslant k<n u N>0$. Тогда при всех $0<\sigma<\infty$

$$
e_{2}(n, k, \sigma, N)=\left(\frac{\widehat{h}_{N}^{2 n-2 k-1}}{\pi} \int_{0}^{\sigma \widehat{h}_{N}} \frac{x^{2(k+n)}}{\left(1+x^{2 n}\right)^{2}} d x+\frac{\sigma^{-(2 n-2 k-1)}}{\pi(2 n-2 k-1)}\right)^{1 / 2},
$$

əдe

$$
\widehat{h}_{N}=\left\{\begin{array}{ll}
h_{N}, & 0<N<\widehat{N} ; \\
0, & N \geqslant \widehat{N},
\end{array} \widehat{N}=\frac{\sigma^{k+1 / 2}}{\pi \sqrt{2(2 k+1)}},\right.
$$

a $h_{N}$ - решение уравнения (17). При этом функиионал

$$
\left\langle\widehat{y}^{*}, F x(\cdot)\right\rangle=\frac{1}{2 \pi} \int_{|t|<\sigma} \frac{(i t)^{k}}{1+\left(\widehat{h}_{N} t\right)^{2 n}} y(t) d t
$$

является әкстремальным. При $\sigma=\infty$

$$
e_{2}(n, k, \infty, N)=\frac{\sqrt{2 k+1}}{\left(4 n^{2} \sin \frac{\pi(2 k+1)}{2 n}\right)^{n /(2 k+1)}}\left(\frac{2 n-2 k-1}{2 \pi N^{2}}\right)^{(2 n-2 k-1) /(2(2 k+1))},
$$

а функиионал (18), в котором

экстремальный.

$$
\widehat{h}_{N}=\left(\frac{2 n-2 k-1}{8 \pi n^{2} N^{2} \sin \frac{\pi(2 k+1)}{2 n}}\right)^{1 /(2 k+1)}
$$

Отметим, что в случае $\sigma=\infty$ результат, сформулированный в этой теореме, может быть получен из работы [12]. 


\section{§3. Доказательства}

В основе доказательств сформулированных теорем лежат соображения, связанные с обшими принципами теории экстремальных задач. Суть дела заключается в том, что рассматриваемые здесь задачи редуцируются к некоторым выпуклым задачам, для которых необходимым и достаточным условием того, что допустимая точка есть решение данной задачи, является равенство нулю производной (или принадлежность нуля субдифференциалу) функции Лагранжа в этой точке. Такое условие представляет из себя некоторое тождество. С другой стороны, сама задача восстановления является двойственной к указанным выпуклым задачам и поэтому, решив их (т.е. получив нужное тождество), мы, вообше говоря, решаем и двойственную задачу (подробнее о таком подходе к решению различных экстремальных задач см. [13]-[15]). Следующая теорема представляет собой итоговый результат приведенных соображений.

Tеорема 7. Пусть $n, k \in \mathbb{Z}, 0 \leqslant k<n, \quad 0<\sigma \leqslant \infty, \quad \delta>0, \quad 1 \leqslant p \leqslant \infty u$ при всех $x(\cdot) \in X_{p}^{n}$ выполняется равенство

$$
x^{(k)}(0)=\left\langle\widehat{y}^{*}, F x(\cdot)\right\rangle+\lambda \int_{\mathbb{R}} x^{(n)}(t) \overline{\widehat{x}^{(n)}(t)} d t,
$$

где $\widehat{y}^{*}$ - некоторый линейный непрерывный функционал на $L_{p}\left(\Delta_{\sigma}\right), \quad \lambda \in \mathbb{R}_{+}$, a $\widehat{x}(\cdot) \in X_{p}^{n}$ и удовлетворяет следующим условиям:

i) $\|F \widehat{x}(\cdot)\|_{L_{p}\left(\Delta_{\sigma}\right)}=\delta$;

ii) $\left\|\widehat{x}^{(n)}(\cdot)\right\|_{L_{2}(\mathbb{R})}=1$;

iii) $\left\langle\widehat{y}^{*}, F \widehat{x}(\cdot)\right\rangle=\delta\left\|\widehat{y}^{*}\right\|$.

Тогда

$$
E_{p}(n, k, \sigma, \delta)=\lambda+\delta\left\|\widehat{y}^{*}\right\|,
$$

а $\widehat{y}^{*}$ - оптимальный метод восстановления. Кроме того, в задаче Стечкина $\partial$ ля $N=\left\|\widehat{y}^{*}\right\|$

$$
e_{p}(n, k, \sigma, N)=\lambda,
$$

а $\widehat{y}^{*}$ - экстремальный функционал.

ДокАЗАТЕЛЬСТво. Учитывая равенство (19), имеем

$$
\leqslant \sup _{x(\cdot) \in C_{p}^{n}}\left|x^{(k)}(0)-\left\langle\widehat{y}^{*}, F x(\cdot)\right\rangle\right|+\delta\left\|\widehat{y}^{*}\right\|=\lambda+\delta\left\|\widehat{y}^{*}\right\| .
$$

С другой стороны, в силу і) для любого метода восстановления $\varphi(y(\cdot))$ имеем

$$
\begin{aligned}
2\left|\widehat{x}^{(k)}(0)\right| \leqslant & \left|\widehat{x}^{(k)}(0)-\varphi(0)\right|+\left|-\widehat{x}^{(k)}(0)-\varphi(0)\right| \\
\leqslant & \sup _{\substack{x(\cdot) \in C_{p}^{n}, y(\cdot) \in L_{p}\left(\Delta_{\sigma}\right) \\
\|F x(\cdot)-y(\cdot)\|_{L_{p}\left(\Delta_{\sigma}\right)} \leqslant \delta}}\left|x^{(k)}(0)-\varphi(y(\cdot))\right| .
\end{aligned}
$$


Отсюда следует, что $E_{p}(n, k, \sigma, \delta) \geqslant\left|\widehat{x}^{(k)}(0)\right|$. Учитывая (19), ii) и iii), получаем

$$
E_{p}(n, k, \sigma, \delta) \geqslant\left|\widehat{x}^{(k)}(0)\right|=\left|\left\langle\widehat{y}^{*}, F \widehat{x}(\cdot)\right\rangle+\lambda\left\|\widehat{x}^{(n)}(\cdot)\right\|_{L_{2}(\mathbb{R})}\right|=\lambda+\delta\left\|\widehat{y}^{*}\right\| .
$$

Из этого неравенства и (21) вытекает равенство (20) и оптимальность метода $\widehat{y}^{*}$.

Перейдем к задаче Стечкина. По доказанному в задаче оптимального восстановления среди оптимальных методов существует метод, задаваемый линейным непрерывным функционалом, поэтому

$$
\begin{aligned}
E_{p}(n, k, \sigma, \delta) & =\inf _{N>0} \inf _{\left\|y^{*}\right\| \leqslant N} \sup _{\substack{x(\cdot) \in C_{p}^{n}, y(\cdot) \in L_{p}\left(\Delta_{\sigma}\right) \\
\|F x(\cdot)-y(\cdot)\|_{L_{p}\left(\Delta_{\sigma}\right)} \leqslant \delta}}\left|x^{(k)}(0)-\left\langle y^{*}, y(\cdot)\right\rangle\right| \\
& \leqslant \inf _{\left\|y^{*}\right\| \leqslant N} \sup _{x(\cdot) \in C_{p}^{n}}\left|x^{(k)}(0)-\left\langle y^{*}, F x(\cdot)\right\rangle\right|+\delta N=e_{p}(n, k, \sigma, N)+\delta N .
\end{aligned}
$$

Следовательно, при всех $N>0$

$$
e_{p}(n, k, \sigma, N) \geqslant E_{p}(n, k, \sigma, \delta)-\delta N
$$

Отсюда из (20) для $N=\left\|\widehat{y}^{*}\right\|$ получаем

$$
e_{p}(n, k, \sigma, N) \geqslant \lambda
$$

С другой стороны, в силу (19)

$$
e_{p}(n, k, \sigma, N) \leqslant \sup _{x(\cdot) \in C_{p}^{n}}\left|x^{(k)}(0)-\left\langle\widehat{y}^{*}, F x(\cdot)\right\rangle\right|=\lambda .
$$

ДокаЗАТЕЛЬСтво теоремы 1 . Докажем, что для всех $x(\cdot) \in X_{\infty}^{n}$ имеет место равенство

$$
x^{(k)}(0)=\frac{1}{2 \pi} \int_{|t|<\sigma_{0}}(i t)^{k}\left(1-\delta \lambda|t|^{2 n-k}\right) F x(t) d t+\lambda \int_{\mathbb{R}} x^{(n)}(t) \overline{\widehat{x}^{(n)}(t)} d t,
$$

где функция $\widehat{x}(\cdot) \in X_{\infty}^{n}$ такова, что

$$
F \widehat{x}(t)= \begin{cases}(-i)^{k} \delta \operatorname{sign} t^{k}, & |t|<\sigma_{0} \\ \frac{(-i)^{k}}{\lambda t^{2 n-k}}, & |t| \geqslant \sigma_{0} .\end{cases}
$$

В силу теоремы Планшереля имеем

$$
\int_{\mathbb{R}} x^{(n)}(t) \overline{\widehat{x}^{(n)}(t)} d t=\frac{1}{2 \pi} \int_{\mathbb{R}} t^{2 n} F x(t) \overline{F \widehat{x}(t)} d t .
$$


Поэтому

$$
\begin{aligned}
& \frac{1}{2 \pi} \int_{|t|<\sigma_{0}}(i t)^{k}\left(1-\delta \lambda|t|^{2 n-k}\right) F x(t) d t+\lambda \int_{\mathbb{R}} x^{(n)}(t) \overline{\widehat{x}^{(n)}(t)} d t \\
& =\frac{1}{2 \pi} \int_{|t|<\sigma_{0}}\left((i t)^{k}\left(1-\delta \lambda|t|^{2 n-k}\right)+\lambda t^{2 n} i^{k} \delta \operatorname{sign} t^{k}\right) F x(t) d t \\
& \quad+\frac{1}{2 \pi} \int_{|t| \geqslant \sigma_{0}}(i t)^{k} F x(t) d t=\frac{1}{2 \pi} \int_{\mathbb{R}}(i t)^{k} F x(t) d t=x^{(k)}(0) .
\end{aligned}
$$

Равенство $\left\|\widehat{x}^{(n)}(\cdot)\right\|_{L_{2}(\mathbb{R})}=1$ легко проверяется. Докажем, что справедливо равенство $\|F \widehat{x}(\cdot)\|_{L_{\infty}\left(\Delta_{\sigma}\right)}=\delta$. При $\sigma_{0} \geqslant \sigma$ это непосредственно вытекает из определения $F \widehat{x}(\cdot)$. Пусть $\sigma_{0}<\sigma$. Тогда $\sigma_{0}=\widehat{\sigma}$ и нетрудно убедиться, что $\left(\lambda \widehat{\sigma}^{2 n-k}\right)^{-1}=\delta$. Тем самым $|F \widehat{x}(t)| \leqslant \delta$ при $|t| \geqslant \widehat{\sigma}$. Проверим теперь выполнение условия іiі) теоремы 7 . Имеем

$$
\left\langle\widehat{y}^{*}, F \widehat{x}(\cdot)\right\rangle=\frac{\delta}{2 \pi} \int_{|t|<\sigma_{0}}|t|^{k}\left(1-\delta \lambda|t|^{2 n-k}\right) d t .
$$

Докажем, что $1-\delta \lambda|t|^{2 n-k}>0$ при $|t|<\sigma_{0}$. В силу определения $\sigma_{0}$ имеем

$$
\delta^{2} \sigma_{0}^{2 n+1} 2(2 n-k) \leqslant \delta^{2} \widehat{\sigma}^{2 n+1} 2(2 n-k)=\pi(2 n+1)(2 n-2 k-1) .
$$

Отсюда вытекает, что

$\delta^{2} \sigma_{0}^{2 n+1}(2 n+1) \leqslant(2 n-2 k-1)\left(\pi(2 n+1)-\delta^{2} \sigma_{0}^{2 n+1}\right)=\sigma_{0}^{-2 n+2 k+1}(2 n+1) \lambda^{-2}$,

т.е. $\delta \lambda \sigma_{0}^{2 n-k} \leqslant 1$. Тем самым при $|t|<\sigma_{0} 1-\delta \lambda|t|^{2 n-k}>1-\delta \lambda \sigma_{0}^{2 n-k} \geqslant 0$. Следовательно, правая часть (25) равна $\delta\left\|\widehat{y}^{*}\right\|$. Для завершения доказательства остается применить теорему 7.

ДоказАтельство теоремы 2. Рассмотрим сначала случай $0<k<n$. Докажем, что для всех $x(\cdot) \in X_{1}^{n}$ имеет место равенство

$$
x^{(k)}(0)=\frac{1}{2 \pi} \int_{\mathbb{R}} \mu_{\delta}(t) F x(t) d t+\lambda \int_{\mathbb{R}} x^{(n)}(t) \overline{\widehat{x}^{(n)}(t)} d t,
$$

где функция $\widehat{x}(\cdot) \in X_{1}^{n}$ такова, что

$$
F \widehat{x}(t)= \begin{cases}0, & |t| \leqslant a \\ (-i)^{k} \frac{|t|^{k}-a^{k}}{\lambda t^{2 n}} \operatorname{sign} t^{k}, & a<|t|<\sigma \\ \frac{(i t)^{k}}{\lambda t^{2 n}}, & |t| \geqslant \sigma .\end{cases}
$$

Действительно, учитывая (24), имеем

$$
\begin{gathered}
\frac{1}{2 \pi} \int_{|t|<\sigma} \mu_{\delta}(t) F x(t) d t+\lambda \int_{\mathbb{R}} x^{(n)}(t) \overline{\widehat{x}^{(n)}(t)} d t=\frac{1}{2 \pi} \int_{|t| \leqslant a}(i t)^{k} F x(t) d t \\
+\frac{1}{2 \pi} \int_{a<|t|<\sigma}\left((i a)^{k} \operatorname{sign} t^{k}+i^{k}\left(|t|^{k}-a^{k}\right) \operatorname{sign} t^{k}\right) F x(t) d t \\
+\frac{1}{2 \pi} \int_{|t| \geqslant \sigma}(i t)^{k} F x(t) d t=\frac{1}{2 \pi} \int_{\mathbb{R}}(i t)^{k} F x(t) d t=x^{(k)}(0) .
\end{gathered}
$$


Непосредственными вычислениями можно убедиться в справедливости равенств

$$
\|F \widehat{x}(\cdot)\|_{L_{1}\left(\Delta_{\sigma}\right)}=\delta, \quad\left\|\widehat{x}^{(n)}(\cdot)\right\|_{L_{2}(\mathbb{R})}=1 .
$$

Остается применить теорему 7.

Пусть теперь $k=0$. Если $\sigma=\infty$, то в силу равенства

$$
x(0)=\frac{1}{2 \pi} \int_{\mathbb{R}} F x(t) d t,
$$

рассмотрев любую функцию $\widehat{x}(\cdot)$, удовлетворяюшую условиям $\|F \widehat{x}(\cdot)\|_{L_{1}(\mathbb{R})}=\delta$, $\left\|\widehat{x}^{(n)}(\cdot)\right\|_{L_{2}(\mathbb{R})}=1$, и применив теорему 7 , получаем утверждение теоремы для $k=0$ и $\sigma=\infty$.

Случай $k=0, \sigma<\infty$ требует отдельного рассмотрения. Для достаточно малых $\varepsilon>0$ определим функцию $\widehat{x}_{\varepsilon}(\cdot) \in X_{1}^{n}$ так, чтобы

$$
F \widehat{x}_{\varepsilon}(t)= \begin{cases}0, & |t| \leqslant \varepsilon \\ \frac{c_{1}}{t^{2 n}}, & \varepsilon<|t|<\sigma \\ \frac{c_{2}}{t^{2 n}}, & |t| \geqslant \sigma .\end{cases}
$$

Положив

$$
\begin{gathered}
c_{1}=\frac{(2 n-1) \delta}{2}\left(\frac{1}{\varepsilon^{2 n-1}}-\frac{1}{\sigma^{2 n-1}}\right)^{-1} \\
c_{2}=\sqrt{2 n-1} \sigma^{n-1 / 2}\left(\pi-\frac{(2 n-1) \delta^{2}}{4}\left(\frac{1}{\varepsilon^{2 n-1}}-\frac{1}{\sigma^{2 n-1}}\right)^{-1}\right)^{1 / 2},
\end{gathered}
$$

нетрудно убедиться, что для функции $\widehat{x}_{\varepsilon}(\cdot)$ выполнены условия $(27)$. Аналогично тому, как это было сделано при доказательстве теоремы 7 , можно показать, что

$$
\begin{aligned}
E_{1}(n, 0, \sigma, \delta) & \geqslant\left|\widehat{x}_{\varepsilon}(0)\right|=\frac{1}{2 \pi} \int_{\mathbb{R}} F \widehat{x}_{\varepsilon}(t) d t \\
& =\frac{1}{2 \pi} \int_{|t|<\sigma} F \widehat{x}_{\varepsilon}(t) d t+\frac{1}{\pi} \int_{\sigma}^{\infty} \frac{c_{2}}{t^{2 n}} d t=\frac{\delta}{2 \pi}+\frac{c_{2}}{\sigma^{2 n-1} \pi(2 n-1)} .
\end{aligned}
$$

Устремляя $\varepsilon$ к нулю, получаем оценку

$$
E_{1}(n, 0, \sigma, \delta) \geqslant \frac{\delta}{2 \pi}+\frac{1}{\sigma^{n-1 / 2} \sqrt{\pi(2 n-1)}} .
$$

С другой стороны, для метода, определенного равенством (12), имеем

$$
\begin{aligned}
& E_{1}(n, 0, \sigma, \delta) \leqslant \sup _{\substack{x(\cdot) \in C_{1}^{n}, y(\cdot) \in L_{1}\left(\Delta_{\sigma}\right) \\
\|F x(\cdot)-y(\cdot)\|_{L_{1}\left(\Delta_{\sigma}\right)} \leqslant \delta}}\left|x(0)-\frac{1}{2 \pi} \int_{|t|<\sigma} y(t) d t\right| \\
& \leqslant \sup _{x(\cdot) \in C_{1}^{n}}\left|x(0)-\frac{1}{2 \pi} \int_{|t|<\sigma} F x(t) d t\right|+\frac{\delta}{2 \pi}=\frac{\delta}{2 \pi}+\sup _{x(\cdot) \in C_{1}^{n}} \frac{1}{\pi} \int_{\sigma}^{\infty}|F x(t)| d t \\
& \leqslant \frac{\delta}{2 \pi}+\frac{1}{\pi} \sqrt{\int_{\sigma}^{\infty} t^{2 n}|F x(t)|^{2} d t} \sqrt{\int_{\sigma}^{\infty} \frac{d t}{t^{2 n}}} \leqslant \frac{\delta}{2 \pi}+\frac{1}{\sigma^{n-1 / 2} \sqrt{\pi(2 n-1)}} .
\end{aligned}
$$


ДОКАЗАТЕЛЬСТВО ТЕОРЕМЫ 3 Проводится по той же схеме, что и теорем 1 и 2 , используя тождество

$$
x^{(k)}(0)=\frac{1}{2 \pi} \int_{|t|<\sigma} \frac{(i t)^{k}}{1+\left(h_{\delta} t\right)^{2 n}} F x(t) d t+\lambda \int_{\mathbb{R}} x^{(n)}(t) \overline{\widehat{x}^{(n)}(t)} d t,
$$

в котором

$$
\lambda=\frac{h_{\delta}^{2 n-k-1 / 2}}{\delta}\left(2 \int_{0}^{\sigma h_{\delta}} x^{2 k}\left(1+x^{2 n}\right)^{-2} d x\right)^{1 / 2},
$$

а функция $\widehat{x}(\cdot) \in X_{2}^{n}$ такова, что

$$
F \widehat{x}(t)= \begin{cases}\frac{h_{\delta}^{2 n}}{\delta} \frac{(i t)^{k}}{1+\left(h_{\delta} t\right)^{2 n}}, & |t|<\sigma \\ \frac{(-i)^{k}}{\lambda} t^{k-2 n}, & |t| \geqslant \sigma\end{cases}
$$

ДОКАЗАТЕЛЬСТво ТЕОРЕМЫ 4 . Обозначим через $N(\delta)$ норму линейного функционала (4) (как функционала на $\left.L_{\infty}\left(\Delta_{\sigma}\right)\right)$. Имеем

$$
N(\delta)=\frac{1}{\pi}\left(\frac{\sigma_{0}^{k+1}}{k+1}-\delta \lambda \frac{\sigma_{0}^{2 n+1}}{2 n+1}\right)
$$

где

$$
\begin{aligned}
& \sigma_{0}= \begin{cases}\sigma, & 0<\delta \leqslant \delta_{0} \\
\left(\frac{\pi(2 n+1)(2 n-2 k-1)}{2 \delta^{2}(2 n-k)}\right)^{1 /(2 n+1)}, & \delta>\delta_{0},\end{cases} \\
& \delta_{0}=\sigma^{-n-1 / 2 \sqrt{\frac{\pi(2 n+1)(2 n-2 k-1)}{2(2 n-k)}}} .
\end{aligned}
$$

Тем самым, учитывая (5), при $0<\delta \leqslant \delta_{0}$

$$
N(\delta)=\frac{\sigma^{k+1}}{\pi}\left(\frac{1}{k+1}-\frac{\delta\left(\pi / \sigma^{2 n+1}-\delta^{2} /(2 n+1)\right)^{-1 / 2}}{(2 n+1) \sqrt{2 n-2 k-1}}\right) .
$$

Нетрудно убедиться, что при $0<\delta \leqslant \delta_{0}$ функция $N(\delta)$ монотонно убывает от $N_{2}$ до $N_{1}$, где

$$
N_{2}=\frac{\sigma^{k+1}}{\pi(k+1)}, \quad N_{1}=\frac{\sigma^{k+1}(2 n-k)}{\pi(k+1)(2 n+1)} .
$$

Следовательно, при $N_{1} \leqslant N<N_{2}$ уравнение $N(\delta)=N$ имеет единственное решение

$$
\delta_{N}=\sigma^{-n-1 / 2} \sqrt{\frac{\pi(2 n+1)(2 n-2 k-1)}{(2 n+1) \gamma^{-2}(\sigma, N)+2 n-2 k-1}} .
$$

При этом с учетом того факта, что при $N_{1} \leqslant N<N_{2} \sigma_{N}=\sigma$, из теоремы 7 вытекает, что экстремальный функционал будет иметь вид (15). 
Если $\delta \geqslant \delta_{0}$, то

$$
N(\delta)=\left(\frac{\pi(2 n+1)(2 n-2 k-1)}{2 \delta^{2}(2 n-k)}\right)^{(k+1) /(2 n+1)} \frac{2 n-k}{\pi(k+1)(2 n+1)} .
$$

При $\delta \geqslant \delta_{0}$ функция $N(\delta)$ монотонно убывает от $N_{1}$ до 0 . Отсюда при всех $0<$ $N \leqslant N_{1}$ сушествует единственное решение уравнения $N(\delta)=N$, выражаемое равенством

$$
\delta_{N}=\sqrt{n-k-\frac{1}{2}}\left(\frac{2 n-k}{\pi(2 n+1)}\right)^{(2 n-k) /(2 k+2)}\left(\frac{1}{(k+1) N}\right)^{(2 n+1) /(2 k+2)} .
$$

При $0<N \leqslant N_{1} \sigma_{N}=\widehat{\sigma}_{N}$ и экстремальный функционал снова имеет вид (15). Выражение для $e_{\infty}(n, k, \sigma, N)$ при $0<N<N_{2}$ получается в соответствии с теоремой 7 подстановкой в $(5) \delta=\delta_{N}$.

Пусть теперь $N \geqslant N_{2}$. Тогда из (22) следует, что при всех $\delta>0$

$$
e_{\infty}(n, k, \sigma, N) \geqslant E_{\infty}(n, k, \sigma, \delta)-\delta N .
$$

Устремляя $\delta$ к нулю, получаем

$$
e_{\infty}(n, k, \sigma, N) \geqslant \frac{1}{\sigma^{n-k-1 / 2} \sqrt{\pi(2 n-2 k-1)}} .
$$

Непосредственная оценка функционала (15), который при $N \geqslant N_{2}$ принимает вид

$$
\left\langle\widehat{y}^{*}, F x(\cdot)\right\rangle=\frac{1}{2 \pi} \int_{|t|<\sigma}(i t)^{k} F x(t) d t
$$

(норма его равна $N_{2}$ ), дает

$$
\begin{aligned}
\mid x^{(k)}(0) & -\left.\left\langle\widehat{y}^{*}, F x(\cdot)\right\rangle\left|\leqslant \frac{1}{2 \pi} \int_{|t| \geqslant \sigma}\right| t\right|^{k}|F x(t)| d t=\frac{1}{2 \pi} \int_{|t| \geqslant \sigma}|t|^{n}|F x(t)||t|^{k-n} d t \\
& \leqslant \frac{1}{2 \pi} \sqrt{\int_{|t| \geqslant \sigma}|t|^{2 n|F x(t)|^{2} d t}} \sqrt{\int_{|t| \geqslant \sigma}|t|^{2 k-2 n} d t} \\
& \leqslant \frac{1}{\sigma^{n-k-1 / 2 \sqrt{\pi(2 n-2 k-1)}}}
\end{aligned}
$$

ДокАЗАТЕЛЬСТво ТЕОРемЫ 5 . Пусть $k>0,0<\sigma<\infty, \varepsilon \in(1,+\infty)$, а $\delta$ определено равенством (9). Обозначим через $N(\varepsilon)$ норму линейного функционала (10) (как функционала на $L_{1}\left(\Delta_{\sigma}\right)$ ). Имеем

$$
N(\varepsilon)=\frac{\sigma^{k}}{2 \pi \varepsilon^{k}} .
$$

Поэтому при $0<N<\sigma^{k} /(2 \pi)$ с учетом тождества (26) утверждение теоремы непосредственно следует из теоремы 7 . Если $N \geqslant \sigma^{k} /(2 \pi)$, то из (22) следует, что при всех $\delta>0$

$$
e_{1}(n, k, \sigma, N) \geqslant E_{1}(n, k, \sigma, \delta)-\delta N .
$$


Устремляя $\varepsilon$ к единище (при этом $\delta \rightarrow 0$ ), получаем

$$
e_{1}(n, k, \sigma, N) \geqslant \frac{1}{\sigma^{n-k-1 / 2} \sqrt{\pi(2 n-2 k-1)}} .
$$

При $N \geqslant \sigma^{k} /(2 \pi)$ функционал (16) принимает вид (28). Учитывая оценку (29), имеем

$$
e_{1}(n, k, \sigma, N) \leqslant \frac{1}{\sigma^{n-k-1 / 2} \sqrt{\pi(2 n-2 k-1)}} .
$$

При $k>0$ и $\sigma=\infty$ норма линейного функционала (10) равна

$$
N(\delta)=\frac{1}{2 \pi}\left(\frac{2 \pi(2 n-2 k-1)}{\delta^{2}(2 n-1)(2 n-k-1)}\right)^{k /(2 n-1)} .
$$

Поэтому при всех $N>0$ найдется $\delta_{N}$, для которого $N\left(\delta_{N}\right)=N$, и с учетом тождества (26) утверждение теоремы вытекает из теоремы 7.

Пусть теперь $k=0$. Тогда при всех $\delta>0$

$$
e_{1}(n, 0, \sigma, N) \geqslant E_{1}(n, 0, \sigma, \delta)-\delta N=\left(\frac{1}{2 \pi}-N\right) \delta+\frac{1}{\sigma^{n-1 / 2} \sqrt{\pi(2 n-1)}} .
$$

Устремляя $\delta$ к бесконечности, получаем

$$
e_{1}(n, 0, \sigma, N)=\infty
$$

Если $N \geqslant 1 /(2 \pi)$, то, устремляя $\delta$ к нулю, из того же неравенства будем иметь

$$
e_{1}(n, 0, \sigma, N) \geqslant \frac{1}{\sigma^{n-1 / 2} \sqrt{\pi(2 n-1)}} .
$$

Обратное неравенство следует из (29) при $k=0$.

ДОКАЗАТЕЛЬСТВО ТЕОРЕМЫ 6 проводится по той же схеме, которая использовалась при доказательствах теорем 4 и 5.

\section{Список литературы}

1. Смоляк C. A. Об оптимальном восстановлении функций и функционалов от них // Дис. ... канд. физ.-матем. наук. М.: МГУ, 1965.

2. Micchelli C.A., Rivlin T.J. A survey of optimal recovery // Optimal estimation in approximation theory / ed. C.A. Micchelli, T.J. Rivlin. New York: Plenum Press, 1977. P. 1-54.

3. Traub J. F., Woźniakowski H. A general theory of optimal algorithms. New York: Academic Press, 1980.

4. Micchelli C.A., Rivlin T.J. Lectures on optimal recovery // Numerical analysis, Proc. SERC Summer Sch., Lancaster/Engl., 1984. Berlin: Springer-Verlag, 1984. P. 21-93. (Lecture Notes in Math. V. 1129.)

5. Арестов В. В. Наилучшее восстановление операторов и родственные задачи // Труды МИАН. 1989. Т. 189. С. 3-20. 
6. Магарил-Ильяев Г. Г., Осипенко К. Ю. Об оптимальном восстановлении функционалов по неточным данным // Матем. заметки. 1991. Т. 50. №6. С. 85-93.

7. Osipenko K. Yu. Optimal recovery of analytic functions. Huntington, New York: Nova Science Publ., Inc., 2000.

8. Магарил-Ильяев Г. Г., Осипенко К. Ю. Оптимальное восстановление функций и их производных по коэффициентам Фурье, заданным с погрешностью // Матем. сб. 2002. T. 193. №3. С. 79-100.

9. Магарил-Ильяев Г. Г., Осипенко К. Ю. Оптимальное восстановление функций и их производных по приближенной информации о спектре и неравенства для производных // Функц. анализ и его прилож. 2003. Т. 37. № 3. С. 51-64.

10. Арестов B. В. Приближение неограниченных операторов ограниченньми и родственные экстремальные задачи // УМН. 1996. Т. 51. №6. С. 89-124.

11. Osipenko K. Yu. Optimal recovery of periodic functions from Fourier coefficients given with an error // J. Complexity. 1996. V. 12. P. 35-46.

12. Тайков Л. В. Неравенства типа Колмогорова и наилучшие формулы численного дифференцирования // Матем. заметки. 1968. Т. 4. № 2. С. 233-238.

13. Магарил-Ильяев Г. Г., Тихомиров В. М. О неравенствах для производных колмогоровского типа // Матем. сб. 1997. Т. 188. № 12. С. 73-106.

14. Магарил-Ильяев Г. Г., Тихомиров В. М. Вьпукльй анализ и его приложения. М.: Эдиториал УРСС, 2003.

15. Magaril-Il'yaev G. G., Osipenko K. Yu., Tikhomirov V.M. Optimal recovery and extremum theory // Comput. Methods Funct. Theory. 2002. V. 2. P. 87-112.

Московский государственный институт

Поступила в редакцию радиотехники, электроники и автоматики

26.02 .2004 (технический университет);

МАТИ - Российский государственный технологический

университет им. К.Э. Циолковского

E-mail: georg@magaril.mccme.ru

konst@osipenko.mccme.ru 